\title{
miR-219-5p inhibits proliferation and clonogenicity in chordoma cells and is associated with tumor recurrence
}

\author{
WEI WEI, QIUHANG ZHANG, ZHENLIN WANG, BO YAN, YANJUN FENG and PU LI \\ Department of Otolaryngology, Head and Neck Surgery, Skull Base Surgery Center, \\ Xuanwu Hospital, Capital Medical University, Beijing 100053, P.R. China
}

Received May 6, 2015; Accepted September 16, 2016

DOI: $10.3892 / \mathrm{ol} .2016 .5222$

\begin{abstract}
Chordoma is a rare malignant bone tumor that is usually localized to the skull base, vertebral column and sacrum. The transcription factor brachyury, which is encoded by the $\mathrm{T}$ gene, has a critical role in the development and progression of chordoma, although the mechanisms underlying brachyury regulation remain unclear. The aim of the current study was to identify and characterize microRNAs (miRs) that regulate brachyury expression in chordoma. MicroRNAs that target brachyury were predicted using miRanda and TargetScan. Using reverse transcription-quantitative polymerase chain reaction, miR-219-5p was shown to be significantly downregulated in chordoma tissues and the $\mathrm{U}-\mathrm{CH} 2$ chordoma cell lines. A dual-luciferase reporter assay was used to validate the inhibitory effect of miR-219-5p on brachyury mRNA expression. The expression level of brachyury was downregulated in $\mathrm{U}-\mathrm{CH} 2$ cells following transfection with miR-219-5p mimics and upregulated following transfection with the miR-219-5p inhibitor. The effects of miR-219-5p on the proliferation and clonogenicity of chordoma cells were assessed using cell counting kit-8, EdU and clone formation assays. These in vitro results indicated that miR-219-5p may have an important role in regulating the cell proliferation and clonogenicity of human chordoma cells, potentially by targeting brachyury. Furthermore, the associations between the expression levels of miR-219-5p and various clinicopathological factors were analyzed, and miR-219-5p expression was shown to correlate with tumor extent and recurrence. These results suggested that miR-219-5p functions as a tumor suppressor in chordoma
\end{abstract}

Correspondence to: Professor Qiuhang Zhang, Department of Otolaryngology, Head and Neck Surgery, Skull Base Surgery Center, Xuanwu Hospital, Capital Medical University, 45 Changchunjie Street, Xicheng, Beijing 100053, P.R. China

E-mail: zhangqiuhang330@163.com

Key words: chordoma, miR-219-5p, brachyury, proliferation, clonogenicity, recurrence and, therefore, that miR-219-50 may be a potential target for therapeutic intervention.

\section{Introduction}

Chordoma is a rare malignant bone tumor with an incidence of $\sim 0.1 / 100,000$ individuals (1). It is thought to originate from notochordal remnants and is usually localized to the skull base, vertebral column and sacrum. Despite improvements in therapeutic modalities, the treatment of chordoma remains challenging because of its location and local recurrence (2). Therefore, understanding the molecular mechanisms underlying the development and progression of chordoma is crucial to identify novel therapeutic targets.

Brachyury, which is a transcription factor encoded by the $\mathrm{T}$ gene, is specifically expressed in chordomas and in the notochord (3). T gene duplication is common in familial chordoma, while gain of the $\mathrm{T}$ locus has also been found in sporadic cases $(4,5)$. Silencing of brachyury using short hairpin RNA has been shown to result in decreased cell proliferation and induction of a senescent phenotype $(5,6)$. It is well-documented that brachyury orchestrates several downstream pathways in chordoma; however, the mechanisms underlying brachyury regulation remain unclear (7).

MicroRNAs (miRNAs or miRs) are a class of small, non-coding, single-stranded RNAs that can regulate the process of translation by binding to the 3'-untranslated region (UTR) of mature mRNAs. Importantly, miRNAs have been shown to be involved in tumorigenesis, angiogenesis, invasion and metastasis (8-10). Furthermore, miRNAs may served as oncogenes or tumor suppressors, and have therefore been reported to be dysregulated in various human cancers, including gastric cancer (11), colorectal cancer (12), thyroid cancer (13) and squamous cell carcinoma (14). However, a role for miRNAs in the regulation of brachyury expression in chordoma has yet to be elucidated.

In the present study, it was first demonstrated that miR-219-5p was downregulated in chordoma tissue samples and the U-CH2 cell line. Therefore, whether brachyury is a direct target of miR-219-5p was evaluated. In vitro analyses showed that miR-219-5p inhibited chordoma cell proliferation and clonogenicity by downregulating brachyury. The associations between miR-219-5p expression and various patient clinicopathological factors were further analyzed and it was 
shown that miR-219-5p expression was correlated with the tumor extent and recurrence.

\section{Materials and methods}

Patients and tissue samples. Tissue samples were obtained from 40 patients who had undergone surgery at Xuanwu Hospital, Capital Medical University (Beijing, China) between February 2008 and February 2013. Freshly received tissues from the operating room were immediately frozen with liquid nitrogen. All patients had confirmed pathological diagnosis according to the World Health Organization classification (15). A total of 40 skull base chordoma samples s and 10 non-neoplastic adjacent tissue samples (normal skeletal muscles) were used in accordance with the policies of the hospital's institutional review board. Written informed consent was obtained prior to initiation of the study from all patients. Medical records were reviewed to obtain clinical information for each chordoma case, including age, gender, tumor site, surgical approach and recurrence. The clinical findings of the 40 patients are listed in Table I.

Cell lines and reagents. The human chordoma cell line U-CH2 (ATCC, Manassas, VA, USA) was cultivated in Iscove's Modified Dulbecco's modified Eagle's medium (Thermo Fisher Scientific, Inc., Waltham, MA, USA) and RPMI-1640 medium (HyClone; GE Healthcare Life Sciences, Logan, UT, USA) at a ratio of $4: 1(\mathrm{vol} / \mathrm{vol})$, supplemented with $10 \%$ fetal bovine serum (FBS; Seromed Biochrom, Berlin, Germany). Culture flasks were coated with rat tail type I collagen (BD Biosciences, San Diego, CA, USA) prior to use. The human embryonic kidney 293T, nasopharyngeal carcinoma CNE-2 and laryngeal carcinoma Hep-2 cell lines were obtained from the Institute of Basic Medical Sciences of the Chinese Academy of Medical Sciences (Beijing, China). 293T cells were cultured in RPMI-1640 medium containing 10\% FBS. CNE-2 and Hep-2 cells were cultured in Dulbecco's Modified Eagle's Medium containing 10\% FBS. All cell lines were cultured in a humidified atmosphere at $37^{\circ} \mathrm{C}$ in $5 \% \mathrm{CO}_{2}$.

Bioinformatics analysis. miRNAs targeting the 3'-UTR of brachyury mRNA were predicted using miRanda (http://www.microrna.org/microrna/home.do) and TargetScan (http://www.targetscan.org).

Reverse transcription-quantitative PCR (RT-qPCR). Total RNA was isolated from tissue samples and cell lines using TRIzol reagent (Invitrogen; Thermo Fisher Scientific, Inc.) and was reverse transcribed using the TianScript RT kit (Tiangen Biotech Co., Ltd., Beijing, China). The reverse transcription reaction was performed in a total volume of $14.5 \mu 1$, which included $2.5 \mu \mathrm{l}(1 \mu \mathrm{g})$ total RNA, $2 \mu \mathrm{l} \mathrm{dNTP}$ mix (2.5 mM), $2 \mu \mathrm{l}$ specific reverse transcription primer $(10 \mu \mathrm{M})$ and $8 \mu \mathrm{l}$ RNase-free water. The reaction mixture was initially incubated at $70^{\circ} \mathrm{C}$ for $5 \mathrm{~min}$, followed by incubation on ice for $2 \mathrm{~min}$. The tubes were centrifuged at $1,000 \times \mathrm{g}$ at $4^{\circ} \mathrm{C}$ for $5 \mathrm{sec}$, after which, $4 \mu 1$ 5X First-Strand Buffer, $0.5 \mu \mathrm{l}$ RNasin (40 U/ $/ \mathrm{l})$ and $2 \mu \mathrm{l}$ reverse transcriptase $(200 \mathrm{U} / \mu \mathrm{l})$ were added to a total reaction volume of $21 \mu \mathrm{l}$. The reaction mixture was then incubated at $42^{\circ} \mathrm{C}$ for $50 \mathrm{~min}$, followed by incubation at $95^{\circ} \mathrm{C}$ for 5 min. $\mathrm{qPCR}$ was performed on an ABI PRISM 7500 Sequence Detection system (Applied Biosystems; Thermo Fisher Scientific, Inc.) using SYBR FAST qPCR Master Mix (Kapa Biosystems, Inc., Wilmington, MA, USA), according to the manufacturer's protocol. The PCR conditions were as follows: $95^{\circ} \mathrm{C}$ for $3 \mathrm{~min}, 60^{\circ} \mathrm{C}$ for $20 \mathrm{sec}$; 40 cycles of $95^{\circ} \mathrm{C}$ for $3 \mathrm{sec}, 60^{\circ} \mathrm{C}$ for $20 \mathrm{sec}$; and $95^{\circ} \mathrm{C}$ for $15 \mathrm{sec}, 60^{\circ} \mathrm{C}$ for $15 \mathrm{sec}$ and $95^{\circ} \mathrm{C}$ for $15 \mathrm{sec}$. Each sample was run in triplicate. The relative expression of miRNA compared with U6 was calculated using the $2^{-\Delta \Delta C q}$ method (16). The primer sequences, which were designed by Beijing Microread Genetics, Co., Ltd. (Beijing, China), were as follows: miR-219-5p stem-loop, 5'-GTCGTA TCCAGTGCAGGGTCCGAGGTATTCGCACTGGATACG AAGAATTGC-3'; miR-219-5p forward, 5'-GGACGGTTG ATTGTCCAAAC-3'; common loop reverse, 5'-TCGTATCCA GTGCAGGGTC-3'; U6 forward, 5'-CTCGCTTCGGCAGCA CATATACT-3'; and U6 reverse, 5'-ACGCTTCACGAATTT GCGTGTC-3'.

Dual luciferase report vector construction and assay. The 3'-UTR fragment of brachyury mRNA containing the seed sequence of miR-219-5p was amplified by PCR using $\mathrm{T}$ gene forward and $\mathrm{T}$ gene reverse primers. The PCR product was then digested using Xho I and Not I (Takara Bio, Inc., Tokyo, Japan) and inserted into the pmiR-RB-Report ${ }^{\mathrm{TM}}$ dual-luciferase vector (Guangzhou RiboBio, Co., Ltd., Guangzhou, China), which contains the firefly luciferase gene Luc and Renilla luciferase gene Rluc, to construct the wild-type T-3'UTR (T-WT) reporter vector. The mutant 3'UTR of the T gene, containing mutated miRNA binding sites, was amplified using T-mut forward and T-mut reverse primers prior to splicing by overlap extension PCR. This was then inserted into the vector to construct the mutant T-3'UTR (T-Mut) reporter vector. The following primer sequences were used (Guangzhou RiboBio, Co., Ltd.): T forward, 5'-GATTACTCGAGAGCAGCAAGG CCCAGG-3' and T reverse: 5'-ATTGCGGCCGCGCAT ATTGCGTTTATTTTG-3'; and T-mut forward, 5'-GTAGCC AAACTGTTAGTGCAGAAAGCATTTTCTG-3' and T-mut reverse, 5'-GCTTTCTGCACTAACAGTTTGGCTACTTTG TCAA-3'. Upon reaching $80 \%$ confluence, $\mathrm{U}-\mathrm{CH} 2$ cells were seeded into rat tail type I collagen-coated 96-well plates at a density of 3,000 cells per well (100 $\mu 1$ medium per well) in triplicate. After $24 \mathrm{~h}$ of cultivation, the cells were transiently co-transfected with $100 \mathrm{ng}$ T-WT or T-Mut reporter vectors and $75 \mathrm{nM}$ miR-219-5p mimics or non-target control (NC; Guangzhou RiboBio, Co., Ltd.) using Lipofectamine 2000 reagent (Invitrogen; Thermo Fisher Scientific, Inc.). After 72 h, firefly and Renilla luminescence were measured using a Modulus $^{\mathrm{TM}}$ Microplate Fluorometer (Turner BioSystems, Sunnyvale, CA, USA) with the Dual-Glo ${ }^{\circledR}$ Luciferase Assay system (Promega Corporation, Madison, WI, USA), according to the manufacturer's protocol.

Cell counting kit (CCK)-8 and EdU assays. U-CH2 cells were seeded into rat tail type I collagen-coated 96-well plates at a density of 3,000 cells per well (100 $\mu 1$ medium per well) in triplicate. Subsequently, the cells were transfected with 75 nM miR-219-5p mimics, NC, miR-219-5p inhibitor or NC inhibitor (Guangzhou RiboBio, Co., Ltd.) using Lipofectamine 2000 . After $24 \mathrm{~h}$ of cultivation, viable cells were 
Table I. Clinical features of chordoma patients and relative expression levels of miR-219-5p.

\begin{tabular}{|c|c|c|c|c|c|c|}
\hline $\mathrm{N}$ & Gender & Age (years) & Tumor site & Surgical approach & Initial/recurrent & $\operatorname{miR}$ \\
\hline 1 & $\mathrm{~F}$ & 25 & Clivus and CV & TA & Recurrent & 0.451 \\
\hline 2 & M & 25 & Clivus & EEA & Recurrent & 0.656 \\
\hline 3 & $\mathrm{~F}$ & 41 & Clivus & EEA & Recurrent & 0.600 \\
\hline 4 & $\mathrm{~F}$ & 13 & Clivus and CV & $\mathrm{TA}$ and $\mathrm{OCF}$ & Recurrent & 0.196 \\
\hline 5 & M & 32 & Clivus and SR & $\mathrm{TA}$ & Initial & 0.960 \\
\hline 6 & M & 62 & Clivus & EEA & Initial & 2.646 \\
\hline 7 & $\mathrm{~F}$ & 39 & Clivus & TA & Recurrent & 0.390 \\
\hline 8 & $\mathrm{~F}$ & 55 & Clivus & EEA & Initial & 0.210 \\
\hline 9 & M & 34 & Clivus & EEA & Initial & 0.918 \\
\hline 10 & M & 44 & Clivus & EEA & Initial & 6.881 \\
\hline 11 & $\mathrm{~F}$ & 52 & Clivus & EEA & Initial & 1.879 \\
\hline 12 & M & 19 & Clivus & EEA & Initial & 0.554 \\
\hline 13 & $\mathrm{~F}$ & 15 & Clivus & TA & Initial & 0.230 \\
\hline 14 & M & 50 & Petroclival region & TA & Recurrent & 0.342 \\
\hline 15 & M & 62 & Clivus & EEA & Initial & 1.332 \\
\hline 16 & $\mathrm{M}$ & 43 & Clivus and ASB & EEA & Recurrent & 0.292 \\
\hline 17 & M & 23 & Clivus & EEA & Initial & 1.609 \\
\hline 18 & $\mathrm{~F}$ & 31 & Clivus & TA & Recurrent & 2.154 \\
\hline 19 & $\mathrm{M}$ & 42 & Petroclival region & EEA & Recurrent & 0.662 \\
\hline 20 & M & 33 & Clivus & EEA & Recurrent & 0.380 \\
\hline 21 & $\mathrm{~F}$ & 49 & Clivus & EEA & Recurrent & 0.898 \\
\hline 22 & $\mathrm{~F}$ & 32 & Clivus & EOA & Initial & 1.822 \\
\hline 23 & M & 56 & Clivus & EEA & Initial & 1.589 \\
\hline 24 & M & 51 & Clivus and SR & EEA & Recurrent & 0.820 \\
\hline 25 & $\mathrm{~F}$ & 59 & Clivus & EEA & Initial & 1.398 \\
\hline 26 & $\mathrm{~F}$ & 46 & Clivus and CV & EOA & Initial & 0.853 \\
\hline 27 & M & 69 & Clivus and CV & EOA & Initial & 1.400 \\
\hline 28 & $\mathrm{~F}$ & 42 & Clivus and ASB & EEA & Recurrent & 0.143 \\
\hline 29 & $\mathrm{~F}$ & 16 & Clivus & EEA & Initial & 1.664 \\
\hline 30 & $\mathrm{~F}$ & 72 & Clivus and CV & EOA & Initial & 0.882 \\
\hline 31 & $\mathrm{~F}$ & 60 & Clivus & EEA & Recurrent & 1.526 \\
\hline 32 & $\mathrm{~F}$ & 66 & Clivus & EEA & Initial & 0.151 \\
\hline 33 & M & 43 & Clivus & EEA & Recurrent & 1.058 \\
\hline 34 & M & 22 & Clivus & EEA & Initial & 4.216 \\
\hline 35 & $\mathrm{M}$ & 32 & Clivus & EEA & Initial & 1.211 \\
\hline 36 & M & 42 & Clivus and CV & $\mathrm{EOA}$ and $\mathrm{OCF}$ & Initial & 0.189 \\
\hline 37 & M & 47 & Clivus and ASB & EEA & Recurrent & 0.350 \\
\hline 38 & $\mathrm{~F}$ & 21 & Clivus & EEA & Initial & 2.359 \\
\hline 39 & $\mathrm{~F}$ & 28 & Clivus & EEA & Recurrent & 0.883 \\
\hline 40 & $\mathrm{M}$ & 50 & Clivus & EEA & Initial & 0.418 \\
\hline
\end{tabular}

$\mathrm{CV}$, cervical vertebra; ASB, anterior skull base; SR, sellar region; TA, traditional approach; OCF, occipitocervical fusion; EEA, endoscopic endonasal approach; EOA, endoscopic transoral approach; miR, relative expression level of miR-219-5p.

evaluated daily for 4 days using CCK-8 assays (Dojindo Molecular Technologies, Inc., Kumamoto, Japan), according to the manufacturer's protocol. Briefly, $10 \mu \mathrm{lCCK}-8$ was added to each well and, after $1 \mathrm{~h}$, the optical density (OD) of the samples was measured at an absorbance of $450 \mathrm{~nm}$ using a microplate spectrophotometer (Epoch 2; BioTek Instruments, Winooski, VT, USA). Each transfection was performed in triplicate. The impact of miR-219-5p on U-CH2 cell proliferation was also assessed using the Cell-Light ${ }^{\mathrm{TM}}$ EdU detection kit (Guangzhou RiboBio, Co., Ltd.), according to the manufacturer's instructions. Briefly, at $72 \mathrm{~h}$ following transfection, cells were incubated with $10 \mu \mathrm{M}$ EdU for $2 \mathrm{~h}$ prior to fixation with $4 \%$ paraformaldehyde for $30 \mathrm{~min}$ and permeabilization using $0.5 \%$ Triton X-100 for $10 \mathrm{~min}$. Proliferating cells were 

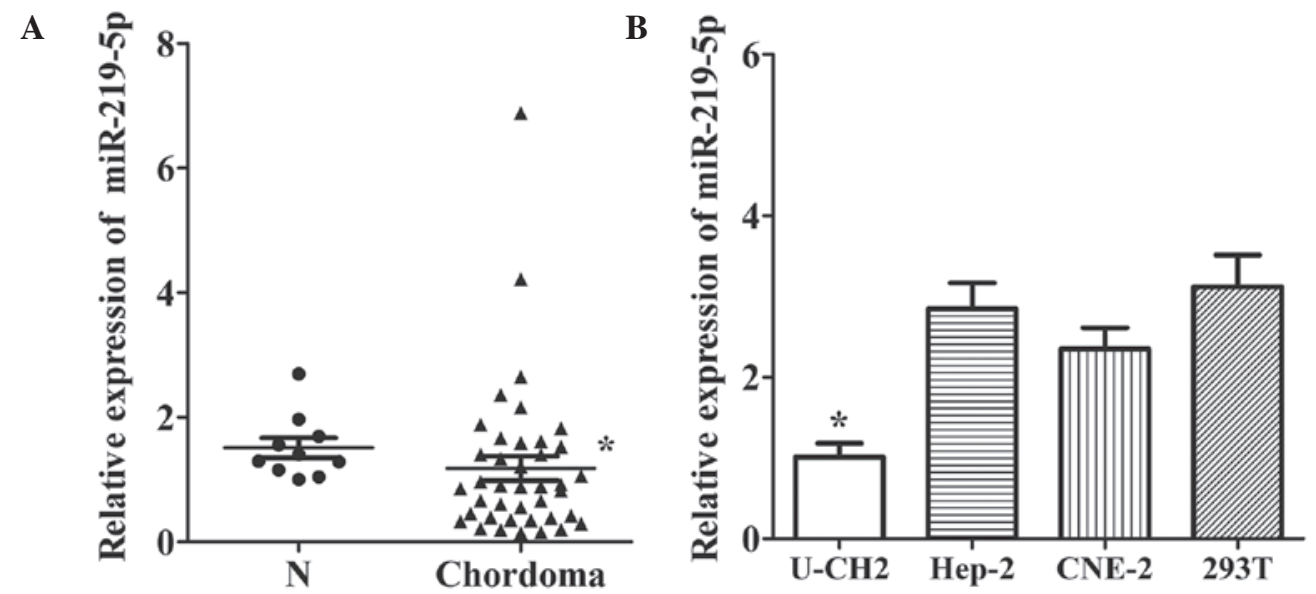

Figure 1. Relative expression of miR-219-5p in chordoma tumor tissues and cell lines. (A) miR-219-5p is downregulated in chordoma tissue samples compared with controls ("P<0.05). (B) Relative expression levels of miR-219-5p in U-CH2, Hep-2, CNE-2 and 293T cell lines ("P<0.01). N, non-neoplastic adjacent tissues.

stained with $100 \mu \mathrm{l} 1 \mathrm{X}$ Apollo643 for $30 \mathrm{~min}$, after which the cells were stained with $100 \mu 11 \mathrm{X}$ Hoechst 33342. Fluorescent cells were counted using the automated Acumen eX3 Laser Scanning Cytometer (TTP Labtech, Ltd., London, UK).

Clone formation assay. U-CH2 or 293T cells were inoculated into rat tail type I collagen-coated 24-well plates at a density of 300 cells per well. After $18 \mathrm{~h}$, the cells were transfected with 75 nM miR-219-5p mimics, NC, miR-219-5p inhibitor or NC inhibitor using Lipofectamine 2000, according to the manufacturer's protocol. Subsequently, the cells were cultured in a humidified chamber at $37^{\circ} \mathrm{C}$ containing $5 \% \mathrm{CO}_{2}$, with culture medium changed every 2 days. After 5 days of cultivation, the cells were stained with $20 \mu \mathrm{M}$ Calcein-AM (Invitrogen; Thermo Fisher Scientific, Inc.) for $45 \mathrm{~min}$. Cell clusters $>200-\mu \mathrm{m}$ in size were considered colonies and were counted using the automated Acumen eX3 Laser Scanning Cytometer.

Western blotting. At $72 \mathrm{~h}$ following transfection, U-CH2 cells were lysed in ice-cold radioimmunoprecipitation assay lysis buffer (Beyotime Institute of Biotechnology, Haimen, China). The protein concentration was estimated using a bicinchoninic acid protein assay (Beyotime Institute of Biotechnology). A total of $50 \mu \mathrm{g}$ protein was separated by $10 \%$ SDS-PAGE and transferred onto polyvinylidene difluoride membranes (EMD Millipore, Billerica, MA, USA). The membranes were blocked with $5 \%$ non-fat dry milk in $0.1 \%$ Tween-20 at $4^{\circ} \mathrm{C}$ for $1 \mathrm{~h}$, then incubated with primary antibodies against brachyury (1:1,000; ab20680; Abcam, Cambridge, MA, USA) or $\beta$-actin (1:2,000; sc-130656; Santa Cruz Biotechnology, Inc., Santa Cruz, CA, USA) overnight at $4^{\circ} \mathrm{C}$. Membranes were washed with TBS and incubated with alkaline phosphatase-conjugated goat anti-rabbit secondary antibody (1:3,000; BA1011; Wuhan Boster Biological Technology, Ltd., Wuhan, China). Respective bands from these blots were observed using the BCIP/NBT Color Development Substrate (Promega Corporation). The experiments were repeated three times. The mean densities of the bands were analyzed using Quantity One 4.6.2 software (Bio-Rad Laboratories, Inc., Hercules, CA, USA) and normalized to that of $\beta$-actin.
Statistical analysis. Statistical analyses were performed using SPSS software, version 21.0 (IBM SPSS, Armonk, NY, USA) and GraphPad Prism, version 5.0 (GraphPad Software, Inc., La Jolla, CA, USA). Differences in miR-219-5p expression were assessed using non-parametric tests (Mann-Whitney $\mathrm{U}$ test) due to the heterogeneity of variances of the residuals when applying analysis of variance. The relative luminescence ratios, ratio of cell proliferation and colony formation, and densities of bands were analyzed using Student's t-tests. Two-sided Fisher's exact test was used to identify differences between categorical variables. $\mathrm{P}<0.05$ was considered statistically significant.

\section{Results}

miR-219-5p is downregulated in chordoma tissues and the $U$-CH2 cell line. Using miRanda and TargetScan, 16 miRNAs targeting brachyury mRNA were predicted, including miR-23a, miR-219-5p, miR-221, miR-222 and miR-448. Of these, RT-qPCR demonstrated that the expression of miR-219-5p was downregulated in chordoma tissue compared with normal skeletal muscle (Fig. 1A). Subsequently, the expression levels of miR-219-5p in U-CH2 cells were compared with CNE-2, Hep-2 and 293T cells (Fig. 1B). These results suggest that the expression of miR-219-5p is significantly decreased in chordoma tissues and the U-CH2 cell line.

miR-219-5p directly binds to the 3'-UTR of brachyury mRNA. The miR-219-5p seed sequence in the 3'-UTR of brachyury mRNA was predicted using miRanda (Fig. 2A). The wild-type and mutant T-3'-UTR fragments, containing the miR-219-5p target site, were inserted into the reporter vectors and validated by DNA sequencing (Fig. 2B). Dual-luciferase reporter assays showed that, when miR-219-5p was overexpressed, the luciferase activity in the wild-type T-3'-UTR reporter vector was significantly reduced (Fig. 2C). However, luciferase activity in the reporter vector containing the mutant T-3'-UTR was not affected by miR-219-5p. These results suggest that miR-219-5p may negatively regulate brachyury expression by directly binding to the GACAAUC sequence in the 3'-UTR of T gene mRNA. 


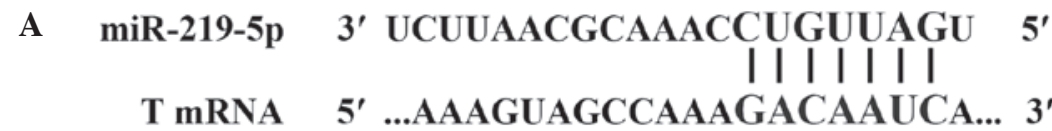

B

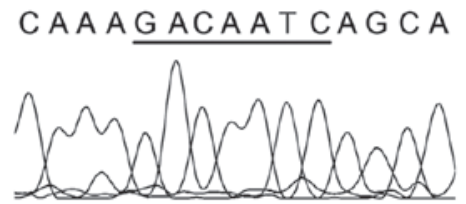

CAAACTGT TAGT GCA

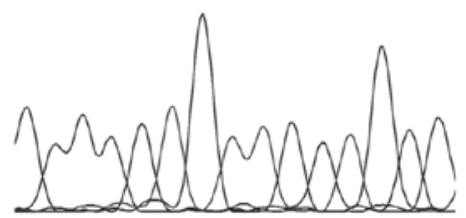

C

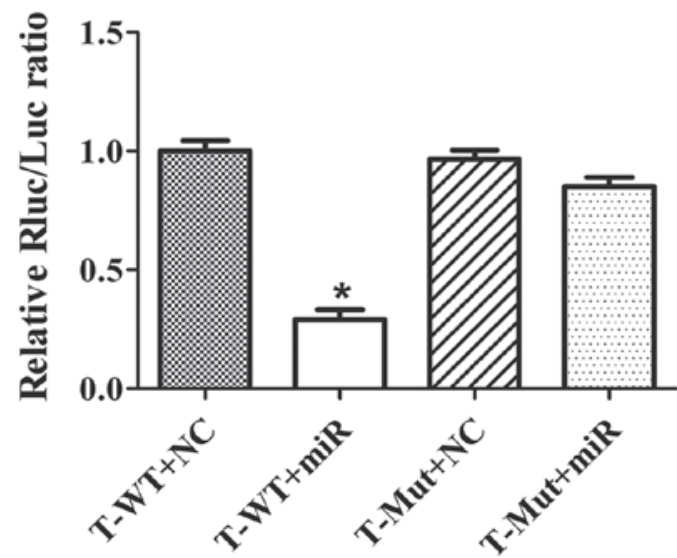

Figure 2. Brachyury is a direct target of miR-219-5p. (A) The binding site of miR-219-5p in the 3'-UTR of the T gene. (B) DNA sequencing of T-WT and T-Mut vectors validated that the binding site of miR-219-5p (GACAATC) was mutated to CTGTTAG in the T-Mut vectors. (C) Dual-luciferase assays confirmed the direct regulation of miR-219-5p on T-3'UTR in U-CH2 cells ('P<0.01). miR, miR-219-5p mimics; 3'-UTR, 3'-untranslated region; T-WT, wild-type T-3'UTR reporter vector; T-Mut, mutant T-3'UTR reporter vector; NC, normal control.

A

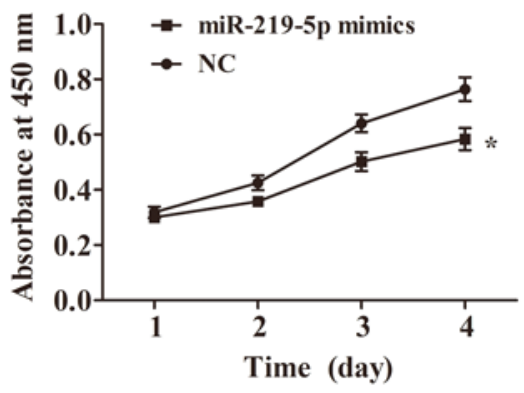

D

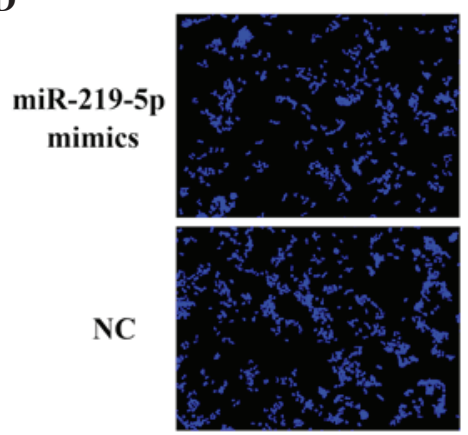

Hoechst 33342
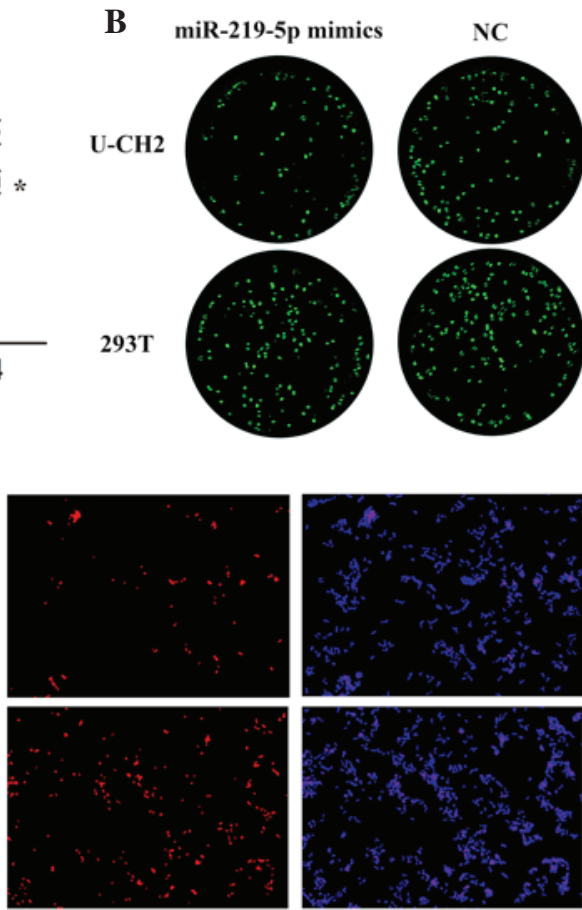

EdU-Apollo643
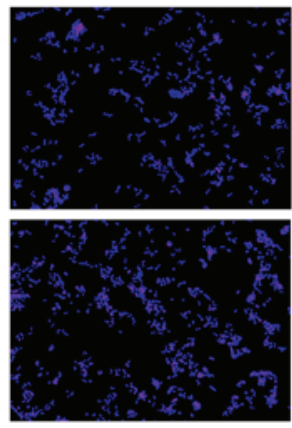

Merge
C

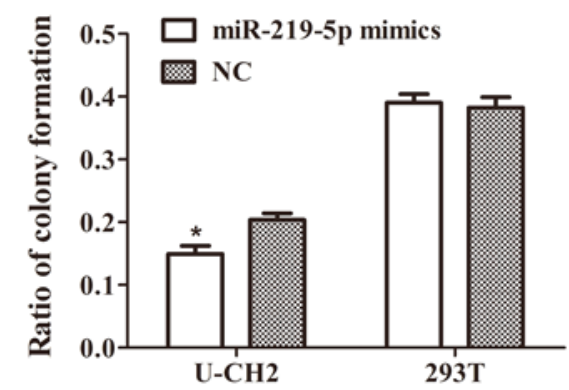

E

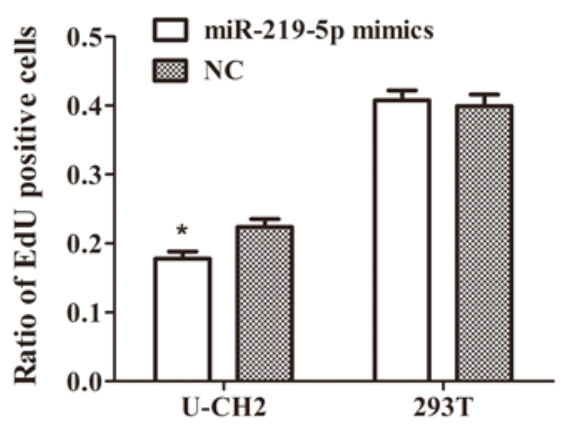

Figure 3. miR-219-5p overexpression suppressed the proliferation and colony formation of chordoma cells. (A) Cell counting kit-8 assays showing reduced cell growth activity in U-CH2 cells transfected with miR-219-5p mimics compared with $\mathrm{NC}$ ("P<0.01). (B) Acumen eX3 laser scanning cytometer imaging showing colonies stained with Calcein-AM (green). (C) miR-219-5p mimics reduced U-CH2 cell colony formation compared with NC, while 293T cell colony formation was unchanged ("P $<0.05$ ). (D) Acumen eX3 laser scanning cytometer imaging showing cells stained with Hoechst 33342 (blue) and proliferating cells labeled with EdU-Apollo643 (red). (E) Proliferation of U-CH2 cells declined following transfection with miR-219-5p mimics compared with NC, while the proliferation of $293 \mathrm{~T}$ cells was unchanged ( $\mathrm{P}<0.05)$. miR, miR-219-5p mimics; NC, normal control.

Effect of miR-219-5p overexpression on cell proliferation and colony formation in chordoma cells. To confirm the biological function of miR-219-5p in chordoma, CCK-8, EdU and clone formation assays of $\mathrm{U}-\mathrm{CH} 2$ cells transfected with
miR-219-5p mimics or NC were performed. As a comparison, the effect of miR-219-5p on proliferation and colony formation was also measured in 293T cells. Significantly reduced cell growth activity $(\mathrm{P}<0.01$; Fig. $3 \mathrm{~A})$, colony formation $(\mathrm{P}<0.05$; 


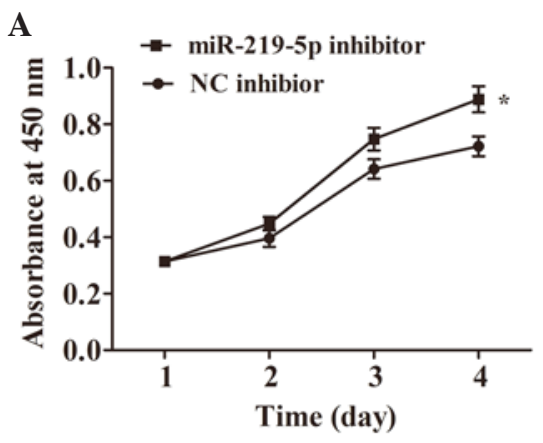

D
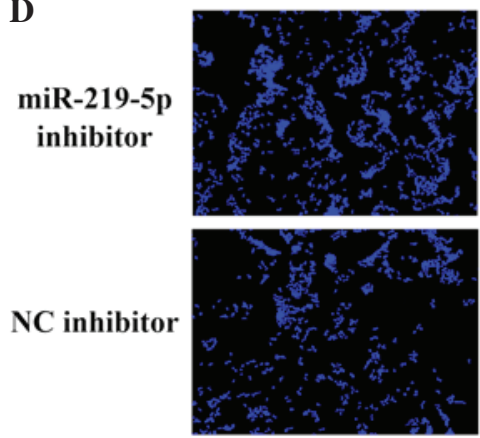

Hoechst 33342
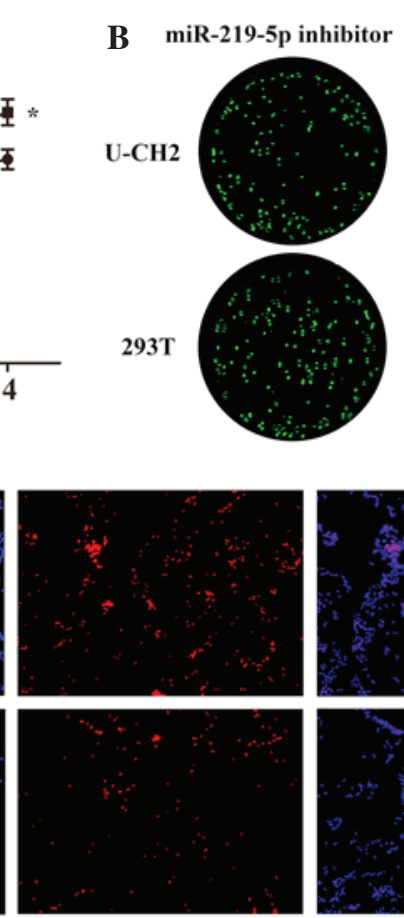

EdU-Apollo643

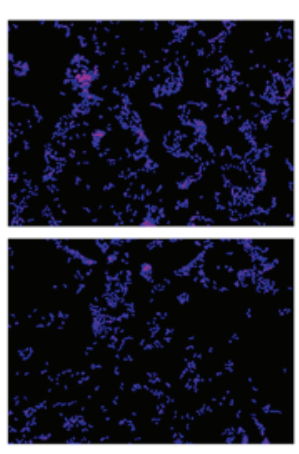

Merge
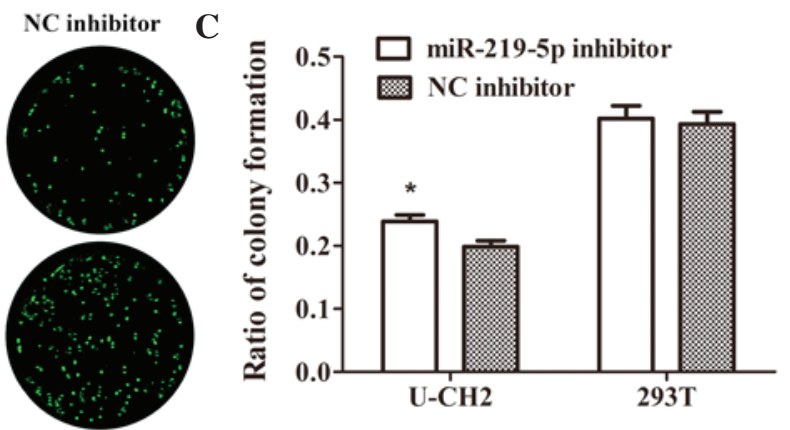

$\mathbf{E}$

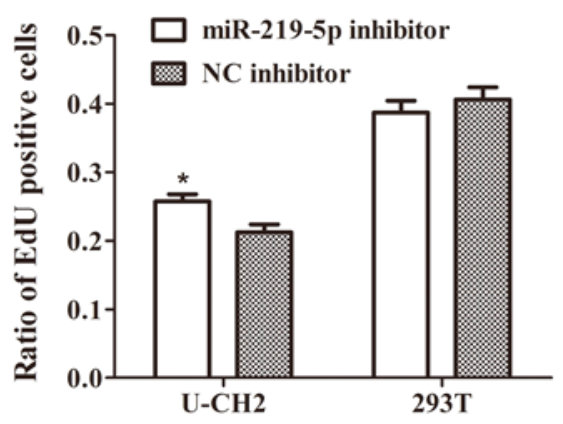

Figure 4. Inhibition of miR-219-5p promoted the cell proliferation and colony formation of U-CH2 cells. (A) Cell counting kit-8 assays demonstrated that transfection with miR-219-5p inhibitor increased U-CH2 cell growth activity compared with NC (*P<0.05). (B) Acumen eX3 laser scanning cytometer imaging showing colonies stained with Calcein-AM (green). (C) miR-219-5p inhibitor increased U-CH2 cell colony formation compared with NC inhibitor, while 293T cells were unaffected ( $\mathrm{P}<0.05$ ). (D) Acumen ex3 laser scanning cytometer imaging showing cells labeled with Hoechst 33342 (blue) and proliferating cells labeled with EdU-Apollo643 (red). (E) Proliferation of U-CH2 cells increased following transfection with miR-219-5p inhibitor compared with NC inhibitor, while $293 \mathrm{~T}$ cells were unaffected $\left({ }^{*} \mathrm{P}<0.05\right)$. NC, normal control.

Fig. 3B and $\mathrm{C}$ ) and proliferation $(\mathrm{P}<0.05$; Fig. 3D and $\mathrm{E})$ were observed in U-CH2 cells transfected with miRNA-219-5p mimics compared with NC. For 293 T cells, there was no significant difference between the cells transfected with miR-219-5p mimics and NC in terms of colony formation (Fig. 3B and C) or cell proliferation (Fig. 3E). These results suggest that overexpression of miR-219-5p is able to suppress cell proliferation and colony formation in $\mathrm{U}-\mathrm{CH} 2$ cells.

Effect of miR-219-5p knockdown on cell proliferation and colony formation in $\mathrm{UCH}-2$ cells. The effect of inhibiting miR-219-5p on U-CH2 and 293T cells was examined. The results showed that downregulating the expression of miR-219-5p by transfection with miR-219-5p inhibitors significantly increased $\mathrm{U}-\mathrm{CH} 2$ cell growth activity $(\mathrm{P}<0.05$; Fig. 4A), colony formation (Fig. 4B and C), and proliferation (Fig. 4D and E), as compared with cells transfected with NC inhibitor. There was no significant difference in cell proliferation or colony formation between the 293T cells transfected with miRNA-219-5p inhibitor and NC inhibitor (Fig. 4). These results indicate that downregulation of miR-219-5p promotes $\mathrm{U}-\mathrm{CH} 2$ cell proliferation and colony formation in vitro.

miR-219-5p regulates the expression of brachyury in $\mathrm{U}-\mathrm{CH} 2$ cells. U-CH2 cells were transfected with miR-219-5p mimic, $\mathrm{NC}, \mathrm{NC}$ inhibitor or miR-219-5p inhibitor, and the effects of miR-219-5p on the protein expression levels of brachyury were analyzed using western blotting (Fig. 5). The results demonstrated that the protein expression levels of brachyury were significantly reduced in miR-219-5p mimic-transfected U-CH2 cells compared with cells transfected with $\mathrm{NC}(\mathrm{P}<0.01)$, but were increased in miR-219-5p inhibitor-transfected U-CH2 cells compared with cells transfected with $\mathrm{NC}$ inhibitor $(\mathrm{P}<0.05)$. These results suggest that miR-219-5p may regulate the protein expression of brachyury in $\mathrm{U}-\mathrm{CH} 2$ cells.

miR-219-5p is correlated with tumor extent and recurrence. Among the 40 patients with skull base chordoma, 23 patients did not receive treatment prior to surgery. Five patients experienced tumor recurrence following the primary surgery and had to be operated on again. Twelve patients underwent the primary surgery at centers other than Xuanwu hospital. There was no significant relationship between the relative expression level of miR-219-5p and patient age $(\mathrm{P}=1.000)$ and gender $(\mathrm{P}=0.755$; Table II). Compared with the initial tumors, the expression levels of miR-219-5p were significantly lower in recurrent tumors $(\mathrm{P}=0.022)$. The expression levels of miR-219-5p were downregulated in 12 of the 13 chordomas where the tumor had gone beyond the clivus. No statistically significant relationship was observed between surgical approaches and the miR-219-5p level $(\mathrm{P}=0.210)$.

\section{Discussion}

Owing to the intricate anatomic location, large tumor size and extensive bleeding during operation, complete surgical resection of chordoma is extremely difficult (2). A better understanding of the molecular mechanisms involved in 
Table II. Correlations between miR-219-5p expression and various clinicopathological characteristics of skull base chordoma.

\begin{tabular}{|c|c|c|c|c|}
\hline \multirow[b]{2}{*}{ Features } & \multicolumn{2}{|c|}{ miR-219-5p (n, \%) } & \multirow[b]{2}{*}{ Total (n) } & \multirow[b]{2}{*}{$\mathrm{P}$-value } \\
\hline & Downregulation $(<1)$ & Upregulation (>1) & & \\
\hline Gender & & & & 0.755 \\
\hline Male & $12(57.1)$ & $9(42.9)$ & 21 & \\
\hline Female & $12(63.2)$ & $7(36.8)$ & 19 & \\
\hline Age (years) & & & & 1.000 \\
\hline$\leq 40$ & $10(58.8)$ & $7(41.2)$ & 17 & \\
\hline$>40$ & 14 (60.9) & $9(39.1)$ & 23 & \\
\hline Tumor site & & & & 0.005 \\
\hline Clivus & $12(44.4)$ & $15(55.6)$ & 27 & \\
\hline Beyond clivus & $12(92.3)$ & $1(7.7)$ & 13 & \\
\hline Initial/recurrent & & & & 0.022 \\
\hline Initial & $10(43.5)$ & $13(56.5)$ & 23 & \\
\hline Recurrent & $14(82.4)$ & $3(17.6)$ & 17 & \\
\hline Surgical approach & & & & 0.210 \\
\hline Endoscopic & $18(54.5)$ & $15(45.6)$ & 33 & \\
\hline Traditional & $6(85.7)$ & $1(14.3)$ & 7 & \\
\hline
\end{tabular}

Data are presented as $\mathrm{n}(\%)$.
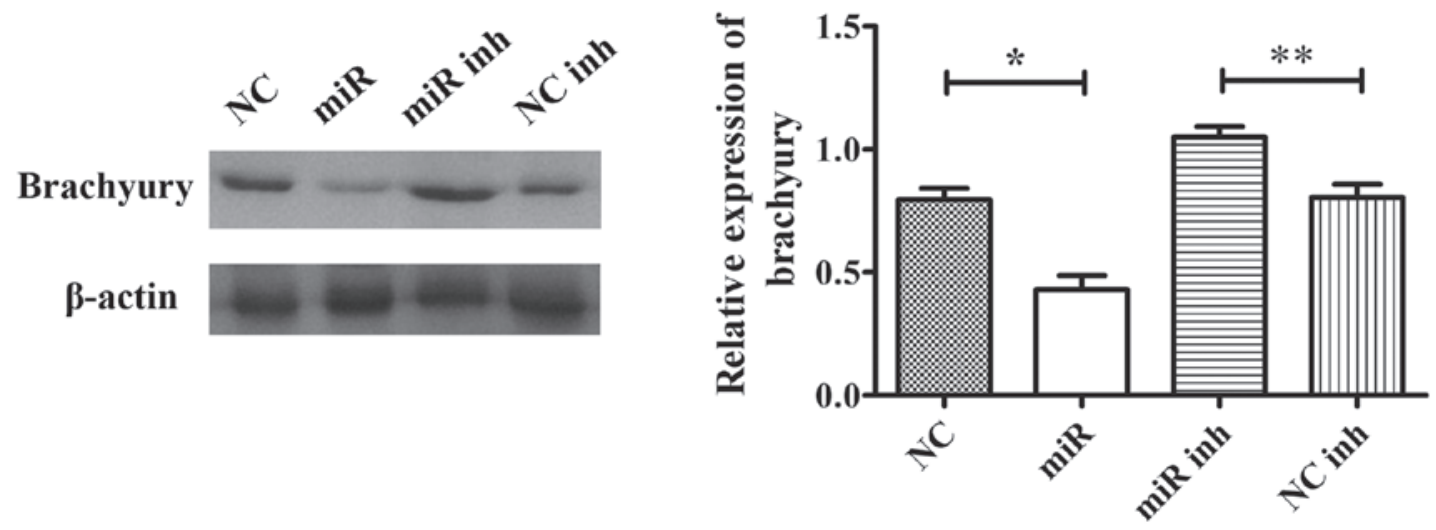

Figure 5. Western blot analysis of brachyury expression in U-CH2 cells followign transfection with miR or miR inh. Brachyury expression was downregulated following transfection with miR ("P<0.01) and upregulated after transfection with miR inh ( $\left.{ }^{* *} \mathrm{P}<0.05\right)$, as compared with $\mathrm{NC}$ and $\mathrm{NC}$ inh, respectively. miR, miR-219-5p mimics; miR inh, miR-219-5p inhibitor; NC inh, NC inhibitor.

tumor progression may assist the development of novel therapeutic strategies for this malignant disease. Accumulating evidence indicates that miRNAs may have an important role in the progression of human chordoma (17-21). For example, reduced expression of miR-1 has been reported in chordoma, and miR-1 is suggested to suppress the growth of chordoma cells by inhibiting Met and histone deacetylase 4 (17). Further studies have shown that miR-1 inhibits chordoma cell migration and invasion and correlates with clinical prognosis $(18,19)$. Another study confirmed that miR-608 and miR-34a were significantly downregulated in chordoma and may influence chordoma malignancy by targeting epidermal growth factor receptor (EGFR), B-cell lymphoma-xL and Met (20). Furthermore, some miRNAs are reported to impact the pathogenesis of chordoma by regulating the mitogen-activated protein kinase (MAPK) signaling pathway (21). In the present study, the relative expression level of miR-219-5p in 40 skull base chordoma specimens and different cell lines were evaluated. Our data suggested that miR-219-5p was downregulated in chordoma tissues and the $\mathrm{U}-\mathrm{CH} 2$ cell line. In addition, the exogenous overexpression of miR-219-5p inhibited the proliferation and clonogenicity of $\mathrm{U}-\mathrm{CH} 2$ cells by targeting brachyury, while suppression of miR-219-5p expression had the opposite effect.

Brachyury is a master regulator in chordoma that binds directly to $\sim 100$ targets and indirectly influences the expression of $>60$ other genes, including genes regulating the cell cycle, growth factors and cytokines, as well as extracellular matrix genes (22). Efforts have been made to clarify the mechanisms underlying the regulation of brachyury in chordoma. In the 
genera Ascidians and Xenopus, as well as zebrafish, brachyury has been shown to be regulated by fibroblastic growth factor receptors via the RAS/RAF/MAPK kinase/extracellular signal-regulated kinase-ETS2 signaling pathway, but genetic alterations of these genes have not been detected in human chordoma (23). The P63 gene can regulate brachyury expression in mouse embryonic fibroblasts and murine-derived osteosarcomas, but not in human chordomas (24). In the present study, the inhibitory effect of miR-219-5p on brachyury, predicted by miRanda and TargetScan, was validated by the dual-luciferase reporter assay. In vitro experiments showed that miR-219-5p regulated the protein expression of brachyury in chordoma cells, and affected cell proliferation and clonogenicity. The results of the present study suggest that the loss of regulation of miR-219-5p on brachyury may be an important mechanism in the initiation and progression of chordoma.

A review of the literature demonstrated that miR-219-5p expression is consistently dysregulated in various tumors (25-29). Downregulation of miR-219-5p has been reported in glioblastoma (25). The exogenous overexpression of miR-219-5p in glioma cell lines could inhibit cell proliferation, growth and migration by targeting EGFR and inhibiting the receptor tyrosine kinase pathway (26). In addition, low expression of miR-219-5p has been associated with higher clinical stages and recurrence rates of meningioma (27). The level of miR-219-5p was reduced in hepatocellular carcinoma. In a previous study, miR-219-5p inhibited cell proliferation in vitro and resulted in the arrest of the cell cycle at the G1/S transition by inhibiting the expression of glypican-3 (28). miR-219-5p was also downregulated in papillary thyroid carcinoma and had a critical role in cell growth by inhibiting estrogen receptor- $\alpha$ (29). These findings, together with the results of the present study, indicate that miR-219-5p may exert a tumor suppressor role in the carcinogenesis of various tumors.

It remains controversial as to whether brachyury is a prognostic indicator for chordoma. Kitamura et al (30) reported that the expression of brachyury was an independent prognostic indicator in skull base chordoma, and was associated with a poor prognosis. Zhang et al (31) analyzed a chordoma tissue microarray and determined that brachyury was not a prognostic indicator for chordoma, although the specimens were mainly derived from mobile spine or sacrum chordoma. Expression of miR-219-5p has been correlated with the recurrence rate of meningioma (27) and overall survival time of hepatocellular carcinoma patients (28). The present study demonstrated that miR-219-5p expression was associated with tumor extent and recurrence, although no definitive conclusion could be drawn owing to the small sample size of the present study. As a regulator of brachyury, the prognostic value of miR-219-5p in skull base chordoma requires further investigation.

In summary, the present study identified that miR-219-5p was downregulated in chordoma tissues and the $\mathrm{U}-\mathrm{CH} 2$ cell line, and was associated with extensive tumor and recurrence. Furthermore, miR-219-5p exhibited the ability to inhibit cell proliferation and clonogenicity of chordoma by targeting brachyury. These findings suggested that miR-219-5p may act as a tumor suppressor in human chordoma, and it may be a potential target for therapeutic intervention in chordoma.

\section{Acknowledgements}

This study was supported by grants from the National Natural Science Foundation of China (grant no. 81170894), the Specialized Project on Scientific Research within the Healthcare Circle by National Health and Family Planning Commission of China (grant no. 201202005) and the Key Discipline Construction Project of the Finance Bureau of Beijing (grant no. 3500-114235).

\section{References}

1. Casali PG, Stacchiotti S, Sangalli C, Olmi P and Gronchi A: Chordoma. Curr Opin Oncol 19: 367-370, 2007.

2. Stippler M, Gardner PA, Snyderman CH, Carrau RL, Prevedello DM and Kassam AB: Endoscopic endonasal approach for clival chordomas. Neurosurgery 64: 268-277, 2009.

3. Vujovic S, Henderson S, Presneau N, Odell E, Jacques TS, Tirabosco R, Boshoff C and Flanagan AM: Brachyury, a crucial regulator of notochordal development, is a novel biomarker for chordomas. J Patho 209: 157-165, 2006.

4. Yang XR, Ng D, Alcorta DA, Liebsch NJ, Sheridan E, Li S, Goldstein AM, Parry DM and Kelley MJ: T (brachyury) gene duplication confers major susceptibility to familial chordoma. Nat Genet 41: 1176-1178, 2009.

5. Presneau N, Shalaby A, Ye H, Pillay N, Halai D, Idowu B, Tirabosco R, Whitwell D, Jacques TS, Kindblom LG, et al: Role of the transcription factor $\mathrm{T}$ (brachyury) in the pathogenesis of sporadic chordoma: A genetic and functional-based study. J Pathol 223: 327-335, 2011.

6. Hsu W, Mohyeldin A, Shah SR, ap Rhys CM, Johnson LF, Sedora-Roman NI, Kosztowski TA, Awad OA, McCarthy EF, Loeb DM, et al: Generation of chordoma cell line JHC7 and the identification of Brachyury as a novel molecular target. J Neurosurg 115: 760-769, 2011.

7. Szuhai K and Hogendoorn PC: 'The chicken or the egg?' dilemma strikes back for the controlling mechanism in chordoma(\#). J Pathol 228: 261-265, 2012.

8. Farazi TA, Spitzer JI, Morozov P and Tuschl T: MiRNAs in human cancer. J Pathol 223: 102-115, 2011.

9. Weis SM and Cheresh DA: Tumor angiogenesis: Molecular pathways and therapeutic targets. Nat Med 17: 1359-1370, 2011.

10. Le XF, Merchant O, Bast RC and Calin GA: The roles of microRNAs in the cancer nvasion-metastasis cascade. Cancer Microenviron 3: 137-147, 2010.

11. Li H, Xie S, Liu M, Chen Z, Liu X, Wang L, Li D and Zhou Y: The clinical significance of downregulation of mir-124-3p, mir-146a-5p, mir-155-5p and mir-335-5p in gastric cancer tumorigenesis. Int J Oncol 45: 197-208, 2014.

12. Xu XM, Qian JC, Deng ZL, Cai Z, Tang T, Wang P, Zhang KH and Cai JP: Expression of miR-21, miR-31, miR-96 and miR-135b is correlated with the clinical parameters of colorectal cancer. Oncol Lett 4: 339-345, 2012.

13. Jikuzono T, Kawamoto M, Yoshitake H, Kikuchi K, Akasu H, Ishikawa H, Hirokawa M, Miyauchi A, Tsuchiya S, Shimizu K and Takizawa T: The miR-221/222 cluster, miR-10b and miR-92a are highly upregulated in metastatic minimally invasive follicular thyroid carcinoma. Int J Oncol 42: 1858-1868, 2013.

14. Ries J, Vairaktaris E, Agaimy A, Kintopp R, Baran C, Neukam FW and Nkenke E: MiR-186, miR-3651 and miR-494: Potential biomarkers for oral squamous cell carcinoma extracted from whole blood. Oncol Rep 31: 1429-1436, 2014.

15. Flanagan AM and Yamaguchi T: Chordoma. In: WHO Classification of Tumours of Soft Tissue and Bone. IARC Press, Lyon, pp.328-329, 2013.

16. Livak KJ and Schmittgen TD: Analysis of relative gene expression data using real-time quantitative PCR and the 2(-Delta Delta C(T)) Method. Methods 25: 402-408, 2001.

17. Duan Z, Choy E, Nielsen GP, Rosenberg A, Iafrate J, Yang C, Schwab J, Mankin H, Xavier R and Hornicek FJ: Differential expression of microRNA (miRNA) in chordoma reveals a role for miRNA-1 in Met expression. J Orthop Res 28: 746-752, 2010.

18. Osaka E, Yang X, Shen JK, Yang P, Feng Y, Mankin HJ, Hornicek FJ and Duan Z: MicroR NA-1 (miR-1) inhibits chordoma cell migration and invasion by targeting slug. J Orthop Res 32: 1075-1082, 2014. 
19. Duan Z, Shen J, Yang X, Yang P, Osaka E, Choy E, Cote G, Harmon D, Zhang Y, Nielsen GP, et al: Prognostic significance of miRNA-1 (miR-1) expression in patients with chordoma. J Orthop Res 32: 695-701, 2014.

20. Zhang Y, Schiff D, Park D and Abounader R: MicroRNA-608 and microRNA-34a regulate chordoma malignancy by targeting EGFR, Bcl-xL and MET. PLoS One 12: e91546, 2014.

21. Long C, Jiang L, Wei F, Ma C, Zhou H, Yang S, Liu X and Liu Z: Integrated miRNA-mRNA analysis revealing the potential roles of miRNAs in chordomas. PLoS One 8: e66676, 2013.

22. Nelson AC, Pillay N, Henderson S, Presneau N, Tirabosco R, Halai D, Berisha F, Flicek P, Stemple DL, Stern CD, et al: An integrated functional genomics approach identifies the regulatory network directed by brachyury (T) in chordoma. J Pathol 228 : 274-285, 2012

23. Shalaby AA, Presneau N, Idowu BD, Thompson L, Briggs TR, Tirabosco R,Diss TCandFlanagan AM: Analysis of the fibroblastic growth factor receptor-RAS/RAF/MEK/ERK-ETS2/brachyury signalling pathway in chordomas. Mod Pathol 22: 996-1005, 2009.

24. Pillay N, Amary FM, Berisha F, Tirabosco R and Flanagan AM: P63 does not regulate brachyury expression in human chordomas and osteosarcomas. Histopathology 59: 1025-1072, 2011.

25. Rao SA, Santosh V and Somasundaram K: Genome-wide expression profiling identifies deregulated miRNAs in malignant astrocytoma. Mod Pathol 23: 1404-1417, 2010.
26. Rao SA, Arimappamagan A, Pandey P, Santosh V, Hegde AS, Chandramouli BA and Somasundaram K: MiR-219-5p inhibits receptor tyrosine kinase pathway by targeting EGFR in glioblastoma. PLoS One 8: e63164, 2013.

27. Zhi F, Zhou G, Wang S, Shi Y, Peng Y, Shao N, Guan W, Qu H, Zhang Y, Wang Q, et al: A microRNA expression signature predicts meningioma recurrence. Int J Cancer 132: 128-136, 2013.

28. Huang N, Lin J, Ruan J, Su N, Qing R, Liu F, Lv C, Zheng D and Luo R: MiR-219-5p inhibits hepatocellular carcinoma cell proliferation by targeting glypican-3. FEBS Lett 23: 884-891, 2012.

29. Huang C, Cai Z, Huang M, Mao C, Zhang Q, Lin Y, Zhang X, Tang B, Chen Y, Wang X, et al: MiR-219-5p modulates cell growth of papillary thyroid carcinoma by targeting estrogen receptor $\alpha$. J Clin Endocrinol Metab 100: E204-E213, 2015.

30. Kitamura Y, Sasaki H, Kimura T, Miwa T, Takahashi S, Kawase $\mathrm{T}$ and Yoshida K: Molecular and clinical risk factors for recurrence of skull base chordomas: Gain on chromosome $2 \mathrm{p}$, expression of brachyury and lack of irradiation negatively correlate with patient prognosis. J Neuropathol Exp Neurol 72: 816-823, 2013.

31. Zhang L, Guo S, Schwab JH, Nielsen GP, Choy E, Ye S, Zhang Z, Mankin H, Hornicek FJ, Duan Z, et al: Tissue microarray immunohistochemical detection of brachyury is not a prognostic indicator in chordoma. PLoS One 8: e75851, 2013. 